\title{
De lo tradicional a lo diverso: la investi- gación creación, un camino para investigar literatura. Apuntes desde la experiencia
}

Fecha de recepción: 04 de abril de 2018

Fecha de aprobación: 18 de noviembre de 2018

\section{Resumen}

La Universidad Pedagógica y Tecnológica de Colombia (UPTC) ofrece, entre sus programas de formación posgradual, el programa de Maestría en Literatura; y este, a su vez, posee una línea de investigación denominada investigación creación. El presente artículo es el resultado de la reflexión que, como estudiante de dicha maestría, tuve en relación con esta metodología de investigación en literatura. Para ello, se hace un recorrido a través de la discusión y los pasos conceptuales que las diferentes instituciones, de validación de conocimiento y de Educación Superior en Colombia, han tomado para definir y legitimar la investigación creación en el ámbito académico. La práctica de la escritura creativa es el resultado de un proceso de investigación.

Palabras clave: Investigación creación, investigación, literatura, escritura, experiencia.

Citar: Chávez Cervantes, A.M. (enero-junio de 2019). De lo tradicional a lo diverso: la investigación creación, un camino para investigar literatura. Apuntes desde la experiencia. La Palabra, (34), 71-83. Got https://doi.org/10.19053/01218530. n34.2019.9529

\section{Ángela María Chávez Cervantes}

Estudiante de la Maestría en Literatura de la Universidad pedagógica y Tecnológica de Colombia. Especialista en Creación Narrativa de la Universidad Central.

angela.chavez@uptc.edu.co

* Artículo de reflexión 


\title{
la palabra
}

\section{From the Traditional to the Diverse: Creative Research as a Path to Investigate Literature, Notes Based on Experience}

\begin{abstract}
The Pedagogical and Technological University of Colombia (UPTC) offers the Masters in Literature as one of its postgraduate programs, and this program has a research line in Creative Research. This article is a result of my reflection on this research methodology as a student of this Masters program. For this purpose, we trace the discussion and conceptual steps taken by different universites and knowledge validation institutions in Colombia to define and legitimize creative research in the Colombian academic context. The practice of creative writing is the result of a research process.
\end{abstract}

Key words: creative research, research, literature, writing, experience

\section{Introducción}

Investigar y crear parecen ir por distintos caminos, debido a una dicotomía que los precede: la discusión y el debate en torno a las llamadas ciencias duras (matemáticas, física y biología) y ciencias blandas (humanidades y las artes). Desde las 1lamadas ciencias duras, se adoptó un modelo de investigación en pro de construir nuevo conocimiento, que hoy en día es considerado tradicional. En este modelo, se proponen objetivos, se construye un camino metodológico para alcanzarlos y se obtienen resultados, medibles, cuantificables y comprobables. Esto se irradió a toda la lógica investigativa en el interior de las universidades. Para unificar los conceptos que se utilizan en investigación, cada disciplina se acogió a una postura epis- temológica, que Pablo Páramo (2013) define como:

El conjunto de suposiciones de carácter filosófico de las que se valen los investigadores, la mayor parte de las veces de forma tácita, para aproximarse al conocimiento [...] la noción que se comparte de realidad y de verdad [...] el papel del investigador en la búsqueda del conocimiento, al igual que la manera como se asume el sujeto estudiado." (p. 24).

El método científico se convirtió en la herramienta de investigación más aceptada y legítima para hacer investigación, y fue relegando otras formas de aproximación al conocimiento. Sin embargo, este método no se ajusta a la postura epistemoló- gica de las artes; por lo tanto, ¿la investigación en artes, también es investigación?

Lo esencial de la cuestión es si existe un fenómeno como la investigación en las artes - según el cual la producción artística es en sí misma una parte fundamental del proceso de investigación, y la obra de arte es el resultado de la investigación. (Borgdorff, 2006, p. 2).

Debido a esta pregunta, en el contexto colombiano surge el debate en torno a la investigación en artes, para entender procesos investigativos en esta área y validar los criterios con los cuales serían evaluados. De manera tran- 
sitoria, estos mecanismos de evaluación de las artes, como productos de conocimiento, han pasado por el ajuste $\mathrm{y}$ la coexistencia con los procesos investigativos en las Ciencias Humanas y en permanente yuxtaposición con la investigación científica.

Uno de los ejemplos más representativos, como lo define Oscar Hernández Salgar, es el de la Facultad de Artes y Humanidades de la Universidad de los Andes, pues en el 2007, hacen explícito el reconocimiento de los productos de creación artística como equiparables con los productos de investigación. Asimismo, en el 2011, la Pontificia Universidad Javeriana abre la primera convocatoria para artistas-investigadores, principalmente en el área de música, para darle lugar a la producción de conocimiento desde la creación.

Posteriormente, la Asociación Colombiana de Facultades y Programas de Artes (Acofartes) construyó un análisis para valorar y legitimar la producción artística en el país. Aunque no fue hasta el año 2013 que, en Colombia, el Departamento Administrativo de Ciencia, Tecnología e Innovación (Colciencias) puso sobre la mesa la posibilidad de la investigación creación y avaló los productos creativos como nuevo conocimiento. Sin embargo, el Ministerio de Cul- tura (2010) ya implementaba "laboratorios de creación" en los que se entendía a la "creación como conocimiento y al conocimiento como creación, con evidentes repercusiones en la construcción y simbolización de las subjetividades personales y colectivas." (p. 1).

Vemos entonces a organismos educativos y sociales buscando la manera de establecer a la investigación creación en el marco de la investigación de las universidades colombianas. Así como quedó registrado en las memorias del evento "Valoración de los procesos de creación artística y cultural en el marco de la acreditación de programas" (2013), a pesar de que la investigación creación no está claramente definida, se está trabajando por definirla, y a su vez, se está dando apertura a espacios donde los estudiantes e investigadores pueden acceder al conocimiento desde una orilla no tradicional.

De la investigación y la creación

Para comprender a la Investigación creación, es necesario cambiar la asociación de conocimiento con la verdad, que nos ha dejado el método científico. La investigación creación hace parte de los nuevos métodos de investigación. Laurel Richardson (1989) propone el proceso investigativo como aquella investigación que se vuelca sobre sí y sobre los sujetos, para reflexionar, dudar $\mathrm{y}$ mostrar que todo lo construido puede ser falseado. Las investigaciones no tradicionales apuntan a lo impuro de las disciplinas, a lo diverso del discurso, a métodos fuera de lo científico para comprender, no para encontrar una verdad develada; dado que la investigación creación no persigue la búsqueda de la verdad, por el contrario, por lo que propende es por el encuentro, la interdisciplinariedad, la construcción individual y colectiva, para otorgarle al conocimiento un espacio de investigación desde lo intuitivo, la imaginación y la sensibilidad.

Durante el proceso de investigación creación, se borran los límites entre el sujeto que investiga y el objeto investigado, puesto que, a la par que se investiga sobre el objeto a crear, se reflexiona sobre la manera en la que se está llevando a cabo y sobre los cambios y transformaciones que tiene el artista al ponerse en contacto con lo que va investigando. Es posible que, a medida que avanza el proceso, el artista-investigador descubra que los aspectos, que en un inicio consideraba relevantes, han dejado de serlo; y, por el contrario, han aparecido va- 
riables importantes que deben ser tenidas en cuenta, como su propia experiencia, su pálpito y su forma particular de organizar las ideas.

Se trata de una indagación que realiza un artista desde su formación disciplinar, su ejercicio profesional $\mathrm{y} / \mathrm{o}$ su experiencia pedagógica; propicia que una práctica artística sea permeada y refundada por el conocimiento reflexivo, a la vez que se compromete a generar referentes conceptuales, teóricos analíticos y creativos que impacten el campo cognitivo, artístico, académico, educativo, productivo, social y/o cultural. (Asprilla, 2015 p. 115).

La investigación creación se convierte en un diálogo de disciplinas y de prácticas culturales, en el que existe un componente social que se mezcla con lo estético y, así, se llega a una puesta en común, donde el cuerpo es pregunta y respuesta de reflexión. El cuerpo de quien investiga hace parte de la investigación: el cuerpo, entendido como una sumatoria de estados, funciones y partes, que unidos se dotan de significado. Dado que, "Explicita una trayectoria creativa y una postura estética personal, por lo cual genera una alta valoración de la subjetividad, la emoción y el pensamiento intuitivo" (Asprilla, 2013, p. $5)$.
Además, como asegura JeanLuc Nancy (2007), el cuerpo siempre está investigando "los vestigios de otro cuerpo". Posee una inspectio mentis, es decir, el cuerpo está dialogando con su interior y esto no se convierte en resultado de investigación, sino en un ejercicio o en una prueba de unas certezas corporales que son susceptibles de transformación. El ser está expuesto en el mundo desde su cuerpo. Esto lo hace ser en el mundo. La exposición del cuerpo en el mundo es la necesidad de comprender el sentido (o la propia lógica) que opera sobre ese cuerpo que se está exponiendo. Por esto, es a partir del cuerpo que se puede hacer investigación creación.

Estas características subjetivas y particulares de la investigación creación, enriquecen el proceso creativo e investigativo, debido a que es necesario el proceso sistemático de búsqueda, clasificación, agrupación y documentación del conocimiento, tanto el aprehendido como el encontrado. No es posible crear sin hacer exploraciones previas, sin contrastar el objeto de la creación con otros similares y con los planteamientos de diferentes disciplinas, o los aportes del colectivo que legitiman y evalúan el resultado obtenido; en el instante en que se unen la investigación y la creación, emerge tanto la obra de arte como el conocimiento y el saber que la produce.

El caso particular de la investigación creación en el campo del lenguaje

En 1981, la Universidad Central crea el primer Taller de escritores (TEUC) en Colombia, este dio paso a la creación de los programas de Especialización en Creación Narrativa (2008), pregrado en Creación Literaria (2010), y la Maestría en Creación Literaria (2012) de la misma universidad. Simultáneamente, en febrero del 2006 nace la Maestría en Escrituras Creativas, de la Universidad Nacional de Colombia; como una forma de profesionalizar el trabajo del escritor, pues, como asegura Azriel Bibliowicz (citado en Orrantia, 2012), su fundador, "la literatura fue desestimulada a toda costa en el Nuevo Mundo. La prohibieron y la persiguieron" (p. 289).

Es curioso que este malestar por incluir la escritura en las universidades, no surgiera del seno de las facultades de literatura. Por el contrario, esta incomodidad por hacer posible la enseñanza de la escritura y validarla profesionalmente, es decir, dejar de ver la literatura como el producto de las musas y la inspiración divina, surgió de los talleres de escritura creativa para convertirse en conocimiento. Que, si bien, su objetivo no apuntaba a 
lo académico, sino a la búsqueda de la expresión, logró introducirse en las universidades.

Martha Orrantia (2012) hace el recorrido por los talleres de escritura creativa del país, que hacían parte del Ministerio de Cultura en el 2009 (Renata), y nos muestra cómo el potencial de estos talleres radicó en la transformación de los sujetos y las comunidades en donde se aplicaban. De allí, su éxito y proliferación tanto en las ciudades como en las provincias.

La experiencia ha sido impresionante. No enseñamos técnicas, porque nosotras no las hemos adquirido. Simplemente hablamos del poder liberador de la literatura y les pedimos que escriban algo, que cuenten su experiencia. Hubo una chica que escribió: 'Hoy llegaron unas cuchas al colegio a hablarnos de la escritura. Yo estaba pensando suicidarme, pero me enseñaron que a lo mejor uno sí puede hacer algo con su vida'. Ese testimonio me alegró porque me mostró que la literatura puede cambiar a la gente. (Orrantia, 2012, p. 294).

Después de estos talleres, las facultades de literatura se preguntan por la posibilidad de enseñar a escribir literatura, y reconocer la escritura como un conocimiento propio del profesional, especialista o magíster de la Literatura (estudios literarios). Ahora, la discusión es casi permanente, debido a que Colciencias aprueba los trabajos de creación como producto de conocimiento.

Dentro de este contexto, está inmersa la Maestría en Literatura de la UPTC y, desde sus inicios, puso sobre la mesa la discusión de la forma de investigar y de allí surge la línea de investigación creación, como una forma de acceder al conocimiento de la literatura desde otro ángulo investigativo. Para esta línea, es necesario e importante, tanto la creación de un producto, como la reflexión que surge y acompaña la creación de ese producto. Pues, siguiendo las ideas de Juliana Borrero y Adrián Farid Freja de la Hoz (2016), esta es la diferencia entre la creación y la creación en la academia. La creación en la academia hace visible el recorrido para construir una obra de arte y, la creación en la universidad se entiende como una forma de conocimiento.

\section{De la escritura creativa a la obra literaria}

La escritura creativa que proponían los talles, mutó a los escenarios académicos y a la creación de literatura; para que esto ocu- rriera, fue necesario pasar por el proceso de evaluación, es decir, que la escritura deja de ser un texto construido en el calor de la emoción y se distancia desde el texto creativo, hacia la construcción artística. Es importante aclarar que, la escritura creativa (en la que el sujeto escribe para representar) y la creación literaria (escritura de literatura), son conceptos distintos; la escritura creativa puede orientarse hacia la técnica, el plasmar ideas o expresar; mientras que la creación literaria apunta a la construcción de nuevo lenguaje, lenguaje artístico y literario. Sin embargo, son conceptos estrechamente relacionados, casi indistinguibles, y, en ocasiones, se aparecen con la misma connotación. Pero, la diferencia sustancial radica en la finalidad de la escritura.

La finalidad artística es la delgada línea que separa la escritura básica de la escritura literaria. Cuando esto sucede en el lenguaje y el habla cotidiana se opera un proceso equiparable con la traducción. Lenguaje y habla ya no dan cuenta de la realidad; sino que se convierten en la herramienta básica para construir, a través de una experiencia y un efecto estético, un nivel de representación bajo la forma de cuento, novela, testimonio, crónica, au-

Juliana Borrero Echeverry, profesional en estudios literarios, escritora, traductora y magíster en Estudios Individualizados Artes Liberales; es docente de la Maestría en Literatura de la Universidad Pedagógica y Tecnológica de Colombia, líder de la línea de investigación creación. Adrián Farid Freja de la Hoz, magíster en Estudios Literarios. Actualmente, cursa sus estudios doctorales. 
tobiografía, ensayo. (Vergara Gamarra, exposición en clase, 19 de octubre 2016).

En literatura, es indispensable el distanciamiento del escritor con su obra, para que sea literatura, y así se cruza la frontera con la escritura representativa y de emociones. En el uso de la investigación creación, para escribir opera de manera simultánea el investigador con el objeto de la creación. Y, durante este proceso, se da la reflexión acerca de para qué y para quién se escribe. "El artista produce la obra en un esfuerzo por escuchar la voz propia como hecho expresivo de su libertad" (Suescún, 2012, p. 41). Dentro del contexto de las universidades, la escritura creativa es la herramienta a través de la cual se mostrarán los resultados de la investigación creación. Puesto que esta se presenta como un texto creativo (inédito) en el cual se hacen evidentes las conceptualizaciones, las argumentaciones y las posturas estéticas que el investigador creador ha tomado, o sea los saberes y conocimientos tanto aprehendidos como descubiertos.

Para que la escritura creativa se convierta en literatura, es necesaria la indagación, que permite al escritor reflexionar y establecer relaciones entre la literatura y los demás productos de la cultura, ya que la escritura no es puramente literaria: está entretejida con otras disciplinas que soportan los temas e ideas plasmados por el escritor. Al asumir la creación dentro de las universidades, y después de ver la discusión que se torna alrededor de este método de investigar, es necesaria la coexistencia con el método tradicional para definir el proceso más allá de los elementos únicos de la investigación-creación.

La investigación-creación en la Maestría de Literatura de la UPTC

Los trabajos de investigación creación, en el caso de mi maestría, están divididos en tres partes: un primer capítulo en el que se analiza un corpus de obras literarias que sirven de "inspiración" para el escrito propio; un texto en donde se evidencie la propia metodología, es decir, un desmontaje de la obra; y, finalmente, la obra. Estos proyectos de investigación creación deben ser aprobados por un magíster en Literatura. Es necesaria la aprobación de un asesor de investigación, exactamente igual que los proyectos de investigación tradicional. Una vez es aprobado por el tutor, el proyecto es llevado a jurados lectores. Por último, es necesaria una sustentación pública del proyecto realizado.

Este tipo de proyectos suscitan un acompañamiento y una evaluación diferente a la tradi- cional. Por ejemplo, un escritor, no exclusivamente profesional en estudios literarios, pero sí magíster; en una ciencia afín, puede ser asesor o evaluador de un proyecto de este tipo. Lo que sí está definido, es que tanto los asesores como evaluadores deben tener conocimientos sobre la investigación creación y sobre la escritura de literatura en la academia.

Actualmente, en Colombia hay pocos profesionales de la Literatura que conozcan los procesos de investigación creación a causa de la reciente inclusión de este método investigativo en las facultades de las universidades colombianas. A mi modo de ver, esto también se debe a que la escritura creativa o la creación literaria, era vista desde las artes o desde los talleres como procesos separados del estudio de la literatura.

Para aprobar este tipo de proyecto, es necesaria la escritura de los tres momentos que mencioné, aunque cabe la pena aclarar que la obra literaria que se construye a lo largo del proyecto, no es un texto acabado. Se trata de un avance de escritura que, en efecto, evidencie la construcción de una voz propia, una postura autoral, un proceso investigativo y una apuesta estética. No en todos los casos es posible que aparezca una obra literaria. 
Los proyectos de investigación tradicional delimitan una problemática que se trata de solucionar. Pero, en la línea de investigación creación de la Maestría en Literatura de la UPTC, se entiende el problema como un problema literario. "Más que una carencia por solucionar, en los trabajos de investigación creación el problema es la declaración de una urgencia por construir a través de la escritura literaria una mirada novedosa; aterrizada en la revisión de obras y trabajos académicos relacionados" (Borrero \& Freja de la Hoz, 2016, p. 18). Estos problemas, vistos así, permiten la inclusión de conceptos teóricos de la literatura y de otras disciplinas, y la revisión cuidadosa de un estado de la cuestión.

El problema de crear una novela, que fue mi caso, era un problema con el lenguaje, con la manera de poner en palabras lo que me imaginaba y pensaba $\mathrm{y}$, más aún, que fuera literatura. Este proyecto, al estar enmarcado en una investigación creación, intentaba buscar el camino para hacerlo. Por un lado, hacer una investigación en torno al tema; y, por el otro, la construcción de una metodología que me permitiera la investigación y, a su vez, desarrollar la escritura creativa.

Luego del problema, aparece la hipótesis (otro elemento del mo- delo tradicional) que se mezcla con la investigación creación; pero, la idea de la hipótesis en investigación creación dista de la que se sugiere en el método científico, dado que, no es una hipótesis que apunte a encontrar la verdad o la certeza de algo, pues, siguiendo las palabras de Silva-Cañaveral (2016), la única certeza de la creación es que no hay certezas.

De lo que sí se hace cargo el arte es de la especulación, incubación y formulación de preguntas de investigación que se relacionan con cuestiones propias de su conocimiento o del ámbito que estudia, en las que se expresan inquietudes y curiosidades de corte estético, creativo, pero también epistemológico. (Silva-Cañaveral, 2016, p. 56)

La formulación de la hipótesis para mi novela, funcionó como guía para no perder de vista lo que quería escribir durante el proceso de escritura, fue mi elección de propuesta estética.

El siguiente paso de la investigación tradicional, es la elaboración de una metodología. En mi caso, utilicé el método-metáfora. Según Márquez (2012), cada escritor debe crear un 'método-metáfora' para su creación, porque cada metodología es única como la obra que produce. Esta metáfora, de una u otra manera, desmonta el proceso creador, es como si al final del cuento se revelara el truco mágico que hizo posible la obra de arte. Es decir, que la investigación creación como forma de investigar contiene en sí mismo variedad de metodologías como de productos que se obtienen. En mi paso por la investigación creación en literatura, este aparte de la investigación era fundamental, pues era la apuesta por teorizar los caminos elegidos para llegar a la reflexión acerca de lo que se estaba construyendo, a la vez que se construía y se contextualizaba desde todas las disciplinas, no solamente la literatura, el valor académico y cognitivo de la creación.

El estado de la cuestión o del arte, para el caso particular de la literatura, también fue necesario, y consistía en hacer el recorrido de creaciones previas, elaborar un corpus de obras literarias que, de una u otra manera, influyen en la creación de un texto nuevo, en tanto que han abordado temas o poseen problemas afines a los que me propuse abordar.

La objetividad es otro elemento del método científico que establece aquello que se estudia fuera del sujeto, y anteriormente, mencioné que en el proceso de investigación creación se desvanecían los bordes que separan al sujeto que investiga con el objeto investigado, porque la escritura es propia e inherente del escritor. Es más, para determinar qué 
es y qué no es literatura, se toma en cuenta al autor de la obra. Las experiencias de la investigación creación que he encontrado vienen en su mayoría del campo de la composición musical, las artes escénicas, no como la mía que es en literatura. Sin embargo, en todos los casos aparece el artista como eje central que produce el sentido de la obra y, por tanto, su involucramiento con su arte hace parte de la investigación, y esto no es distinto en el caso de la literatura.

El arte mismo se exige, en su rigor, dimensionar, conceptuar defender y organizar el enfoque y la participación de esas otras disciplinas para que así se comprenda el método-obra como un proceso, uno mismo e insoluble. Que cada vez nace único y en la experiencia [...] Al tiempo una puesta para que las prácticas de subjetividad, sean un asunto que logre ser comprendido de manera abierta y "sin pedestal" en el escenario social y pedagógico del conocimiento. (Hortua et al., 2011, p. 9).

Retomando el hilo de la delimitación y criterios de evaluación, en las distintas ramas de la investigación artística, cada institución educativa se ha propuesto sus propios criterios de evaluación, en un intento por ajustar la investigación crea- ción en los parámetros de resultados de la investigación tradicional. En mi Maestría, lo que sí es necesario tener en cuenta de la creación de literatura, son: el valor estético y el aporte literario. Aquella experiencia de investigar e investigarse mientras que se escribe, debe tener un valor estético para ser literatura.

De manera que, la escritura no es un objeto extraño, sino que también hace parte de mí; pues, tiene que ver con la postura que toma el investigador- creador y, simultáneamente, aparece el aporte literario. Si se está estudiando literatura y se está investigando sobre la literatura, no importa que no sea con el método tradicional (teoría, historia, crítica), siempre debe haber una contribución al campo de estudio, a la disciplina que se está estudiando. Por supuesto, estos dos criterios de evaluación aplican al producto de la creación literaria y a su respectiva reflexión.

De la escritura de una obra literaria (en proceso) al producto

Por último, en el método tradicional tenemos el proceso de divulgación, que en el caso de la investigación creación en literatura, es la escritura de una obra literaria. Este parece ser el criterio de evaluación más difícil de definir, entre otras cuestiones, porque surge la pregunta sobre si el producto de la investigación creación de literatura lo debe evaluar un crítico literario o un escritor, o los lectores. La obra literaria, como producto de la industria cultural y de mercado, también tiene una valoración al interior de la academia y también es valoradora por los agentes del campo de la literatura.

La obra literaria, producto de un proceso de investigación creación, da cuenta de la investigación y de lo aprendido durante el proceso. El texto final o parcial es el manifiesto del escritor sobre la escritura, la reflexión sobre el lenguaje. La obra literaria que se forma en el seno de la universidad, da cuenta de la forma en que brota la palabra poética y el lenguaje literario. La escritura literaria es lenta, lentísima. La construcción de una voz narrativa puede tardar años, tal vez, una vida en germinar y madurarse, de ahí el principal obstáculo para divulgar a los productos de la investigación creación en literatura como otros productos de divulgación académica.

Análogamente, hay una premisa mayor al hablar de escritura de literatura en la universidad, la escritura también es conocimiento. 
El conocimiento se caracteriza por tener una naturaleza física y, por lo tanto, se puede considerar como un bien, en la medida en que puede circular o ser intercambiado. Así, se puede decidir que para cierto conocimiento considerado como valioso, se reserve su confidencialidad para convertirse en objeto de transacciones económicas y comerciales. (Ballesteros, 2018, p. 22)

El conocimiento visto desde el paradigma positivista de la producción y el consumo masificado, en donde los textos literarios se vuelven objetos desechables y genéricos para las ventas masivas, deforman completamente la autenticidad del producto de la investigación creación de literatura. Esta es una discusión vigente $\mathrm{y}$ actual. Colciencias evalúa el conocimiento a partir de las patentes, los productos tangibles, los impactos en las comunidades, los montajes, las exposiciones, los edificios, las composiciones y, por supuesto, en el caso de la literatura, las publicaciones.

Los productos de la investigación creación, en este caso la novela, los cuentos, los ensayos, etc., se ajustan a los medios y prácticas de producción y difusión del conocimiento, porque "Las áreas creativas, al generar nuevo conocimiento, producen creaciones en las que el aspecto tangible suele ser más evidente que en otras áreas del conocimiento". (Ballesteros, 2018, p. 22) En las universidades, estos productos están dirigidos a un público especializado para su evaluación, pasan por las manos y el reconocimiento de los pares, los profesores, artistas de la misma disciplina, por editores, por premios de la academia: antes de venderlos deben ser aceptados y valorados desde su naturaleza y esencia subjetiva. Siguiendo las palabras del maestro Isaías Peña $(2014)^{2}$, en la universidad se va a aprender de literatura, se va a aprender a escribir, y bueno, gradualmente a publicar. Pero, no para enseñarles a los estudiantes a reproducir fórmulas de libros para las ventas; el paso por la escritura literaria es aprender a ver el mundo con ojos propios, y buscar la voz para expresarlo.

Las universidades que toman el camino $y$, valientemente, le abren la puerta a la escritura creativa, están en permanente construcción de parámetros de evaluación de estos productos y, análogamente, en el proceso de cohabitar el fenómeno de ingresar a la escritura que surge en sus instituciones a los productos de la industria cultural. En mi paso por la línea de investigación creación de la UPTC, he visto a narradores y poetas, pero, por sobre todas las cosas, he visto a personas que leen el mundo, que ven al mundo con ojos de artistas, personas que han sido formadas y transformadas por el lenguaje literario, por la búsqueda de lo indecible del mundo y de sí mismos, he visto cómo investigar y crear ha hecho (maestros) magísteres en literatura. Aunque no todos hayan publicado sus textos, ni sean un éxito en ventas.

El nacimiento mi trabajo de investigación creación en literatura

A continuación, expondré tres pasos metodológicos que seguí en el curso de la escritura de mi trabajo de investigación creación. El primer paso que tomé, como una escritora cachorra, fue el de admirar y desear escribir como los grandes maestros de la literatura, mis escritores favoritos y los elogiados por la academia. Como escritora 'cachorra', quería ser un alquimista de mis gustos y lo que le gusta a la crítica. Primero que todo, surgió la emulación, la mimesis pura, el gesto de imitar o copiar de manera tan perfecta, que fuera imposible distinguir

2 Isaías Peña Gutiérrez, maestro, escritor, periodista, profesor. Fundó en la Universidad Central (Bogotá): el Taller de Escritores (1981), la Especialización en Creación Narrativa (2008) y el pregrado de Creación Literaria (2010). Dirigió allí el Depto. de Humanidades y Letras (hoy, Depto. de Creación Literaria) entre 1997 y 2016. Hoy, dirige el TEUC y la revista Hojas Universitarias, Universidad Central. 
mi voz narrativa de la de los escritores emulados. De todas formas, esto nunca funcionó, aunque implicó un enorme esfuerzo de lectura, y dedicación para escribir. Leer como escritor, pensado en la voz poética, en los recursos lingüísticos, en los trucos que utiliza cada autor para imitar, es un trabajo sistemático, puntilloso y delicado, en el que conscientemente se toman decisiones de corte estético, estructural y formal; por ejemplo, establecer el narrador, crear los personajes, elegir el género literario y definir el argumento.

Durante este paso, surgió la búsqueda para acercarme a la literatura e incluirme en la tradición literaria a cualquier costo; incluso 'plagiando' un texto famoso o no tan famoso, pero, que satisfacía al público, al lector que estaba acostumbrado a leer. Se trataba de apuntarle a lo que ya está hecho, era tomar el camino que ya había sido labrado. Para determinar este afán de insertar la escritura, en el sistema de publicación y ovación de la academia, tomé el camino que Escobar Vera3 define como fósil: "una estructura y unas decisiones estéticas que han sido creadas por otros y repetidas de manera incontable por muchos más, y que siguen satisfaciendo a la crítica literaria y al público". Ese camino carece de todo sentido. No obstante, si no se logra leer de esta manera, si no se logra desmenuzar las obras literarias hasta los huesos y entender cómo funcionan desde las palabras hasta el texto sólido y todos los agentes del campo de la literatura que lo rodean, no es posible crear un texto literario nuevo. Este paso, aunque poco propositivo, es indispensable en la creación, y es el primero.

El segundo paso que tomé para crear mi proyecto fue el rompimiento; esto, en vista de que la creación de una nueva obra no es solo copiar. Descubrí que no todo en literatura es repetición, ni era conocer exhaustivamente la literatura y sus formas. Crear también suponía oposición. Para crear mi novela, fue necesario el rompimiento, la crítica, la posibilidad de subvertir los cánones literarios, la escritura creativa como escenario para transgredir a la tradición literaria y opté por ir en contra de todo lo determinado y establecido. Ir en contra hasta del sentido común, de la verosimilitud, de la métrica, de los géneros, ir en contra de todo. En este paso, me negué a aceptar las opiniones de otros y defendí, a toda costa, mi postura estética, mis decisiones formales de escritura. Esto fue muy importante, pues aquí teoricé y argumenté, construí el diálogo con otras disciplinas que iban soportan- do la creación; me desbocaba investigando, más allá de la literatura, para darle forma a una idea propia. Pero, estaba sorda al colectivo, a mis pares, y esto produjo una creación vacua y contestataria.

En el tercer paso, recuperé el oído. Comencé otra vez. Le aposté a la idea propia, pero, ya no para revelarme o ir en contra; tampoco, para insertarme a toda costa, en la tradición literaria. Es en este paso cuando, de manera consiente, creo que escribí literatura. Era un juego en el que operaban cada uno de los pasos anteriores, dado que necesitaba la lectura rigurosa de los predecesores, el trabajo arduo de recopilar y catalogar lo esencial de cada obra leída. Luego de esto, elegí un camino propio que estuviera sustentado en la literatura y en las otras disciplinas, con el fin de que la obra se articulara con las demás, la crítica, los lectores, el colectivo y conmigo misma.

Uno puede descubrir a los otros en uno mismo, darse cuenta de que no somos una sustancia homogénea, y radicalmente extraña a todo lo que es uno mismo: Yo es otro. Pero los otros también son yos: sujetos como yo, que solo mi punto de vista, para el cual todos están allí y solo

Hernando Escobar Vera, profesor de la maestría en Literatura de la Universidad Pedagógica y Tecnológica de Colombia, comunicador social, magíster en Literatura Hispanoamericana del Instituto Caro y Cuervo, máster en Psicoanálisis y Teorías de la Cultura de la Universidad Complutense de Madrid. Actualmente, cursa sus estudios doctorales 
yo estoy aquí, separa y distingue verdaderamente de mí. (Todorov, 1998, p. 13).

Desde mi experiencia investigando para crear una novela (literatura), solo fue después de pasar por estos tres momentos que comprendí que para escribir es necesario el nacimiento. Peter Sloterdijk (2006) propone la inexistencia de fórmulas para conectarse con el mundo. A partir de esta premisa, plantea el nacimiento como el hacerse a sí mismo, hacerse consciente de su propia existencia y de sus potencialidades. En cuanto a esto, se refiere a "desligarse"; es decir, la posibilidad de encontrase en el interior de un caos, dejarse sorprender y actuar con libertad. Este es el espacio de la escritura, ya que, siguiendo la línea de pensamiento kantiano, nacer es ser autónomo.

Esto es fundamental, puesto que el ser autónomo solo es posible en el decir. El lenguaje es el filtro con el que se interpreta el mundo; ergo, "la promesa del lenguaje", es la posibilidad de ser libre. Y solo en este escenario es posible nacer, tomar conciencia, ir al pasado y volver para construir, para crear algo nuevo. El arte, para Sloterdijk, aparece entonces por la pequeña diferencia de que un "yo" consciente se introduce en el mar de la tradición artística. Es así como buscamos nacer, y nacemos en el lenguaje para encontrar esa "pequeña diferen- cia". En ese instante, fui consciente de mi escritura, y nació mi novela.

\section{Consideraciones finales}

La búsqueda de información acerca de la investigación creación, en Colombia, arroja un panorama de investigación universitaria muy tradicional (hasta anacrónico) y poco diverso. Afortunadamente, estamos en medio del debate y del planteamiento de nuevas formas de investigar el arte de manera más interdisciplinar, más integral, donde los caminos y metodologías sean múltiples.

La investigación creación es solo una propuesta para estudiar el campo de la literatura metodológicamente, con un esquema más flexible y adaptable que el modelo científico, tradicional, en el que hasta ahora se ha estudiado en este campo. Pues, pueden existir más estrategias para aprender y construir nuevo conocimiento, tomando en cuenta a los sujetos, a los artistas, durante el proceso. Han pasado tan solo cinco años desde que Colciencias aprobó la creación como conocimiento producto de la investigación; por consiguiente, queda un extenso camino para seguir definiendo y legitimando a la investigación creación dentro de las universidades, con miras a que oxigene, se invente y se reinvente la investigación colombiana.
La creación es conocimiento, por eso al inicio de este texto planteé la postura del Ministerio de Cultura por encima de algunas universidades, pues esta mirada de la creación, y la creación artística como saber propio de un sujeto y una comunidad, es la que realmente le da sentido y cabida a investigar para crear o crear investigando. Es conocimiento porque no se trata de dominar una técnica ni repetir unos pasos, se trata de buscar una nueva lógica, la que es única y propia de los artistas. Por esto, el artista también hace parte de su obra, en términos de la investigación es una variable que modifica los resultados. Desde la creación, se pueden dar un apretón de manos las antiguas rivalidades entre las ciencias duras y las artes. Para ambas, es necesaria la imaginación, la rigurosidad, y la interacción entre la academia y su contexto, o, mejor dicho, la armonía entre el conocimiento, el sujeto y su comunidad.

Para finalizar, durante este proceso de investigación, pude constatar la presencia del escritor y el involucramiento del mismo sujeto que investiga con el que crea. Yo hago parte de lo que escribo, ya que mi cuerpo está involucrado. Trato de buscar, a medida que escribo, a toda costa, que sea literatura; y me encuentro con unas letras que reflejan mis lecturas, pensamientos, creencias y conocimientos sobre la literatura $\mathrm{y}$, 
con gran felicidad, hallo argumentos lo suficientemente sólidos para convencer a los que me rodean en la universidad.

Asimismo, me encuentro con textos incompletos, difíciles de entender para otros, estos textos son retos que debo resolver y que el camino para hacerlo es seguir investigando, profundizando cada vez más en la literatura para ir forjando mi escritura. Como escritora siempre seré una obra inacaba.

Por esto, y lo dicho anteriormente, en el caso particular de la literatura, las obras que se escriben en la universidad son valiosas e importantes para el campo literario, porque es indispensable la teorización del conocimiento nuevo, tanto de la técnica y la valoración literaria como el conocimiento de sí mismo, de quien escribe. Es valiosísimo para una universidad, para la comunidad y para los sujetos, descubrir paralelamente tanto los saberes formales como los saberes propios de cada cuerpo. La investigación creación es construir y fomentar la seguridad, no la certeza, de los momentos apropiados para escribir, de las formas de traer a la imaginación y a la mente las imágenes del mundo, y de sí mismos, para transformarlas en palabras, en lenguaje y en literatura.

\section{Agradecimientos}

Al Grupo de investigación Senderos del Lenguaje, de la Universidad Pedagógica y Tecnológica de Colombia, en cabeza de Juliana Borrero, profesora, escritora, amiga e investigadora de la literatura, quien desde el cuerpo, y a pesar de las vicisitudes, me llevó por el camino de la investigación creación.

\section{Referencias}

Asprilla, L. I. (2013). El proyecto de creación investigación: la investigación desde las artes. Cali: Instituto Departamental de Bellas Artes.

Asprilla, L. I. (2015). La producción de conocimiento desde las artes: propuesta para un Programa Nacional de Artes adscrito a Colciencias. En Creación, pedagogía y políticas del conocimiento, II encuentro (pp. 105-123). Bogotá: La silueta ediciones.

Ballesteros, M. (2018). ¿Investigar creando?: una guía para la investigación-creación en la academia. Bogotá: Universidad El Bosque, Facultad de Creación y Comunicación.

Borgdorff,H.(2006).Eldebate sobrela investigaciónenlas artes.AmsterdamSchooloftheArts. Recuperado de https://www.researchcatalogue.net/profile/download- media?work=129470\&file=129476

Borrero, J. \& Freja, A. F. (2016). Manual de investigaciones, Lineamientos para el trabajo de grado. Maestría en Literatura. Tunja: Universidad Pedagógica y Tecnológica de Colombia.

Colciencias. Departamento Administrativo de Ciencia, Tecnología e Innovación. (2015). Modelo de medición de grupos de investigación, desarrollo tecnológico o de innovación y de reconocimiento 
de investigadores del Sistema Nacional de Ciencia, Tecnología e Innovación, año 2015. Bogotá: Colciencias.

Hortua, D.F., Márquez, E., Becerra, H., \& Numpaque, G. (2011). Investigación - creación taller y viaje. Tunja: Universidad Pedagógica y Tecnológica de Colombia.

Márquez, E. (2012). Introducción. En Santa Clara la Real, Una casa para Crear: memorias del Laboratorio de Investigación-Creación En-tornos. Tunja: Ministerio de Cultura.

MinCultura. Ministerio de Cultura de Colombia (2010). Laboratorios de investigación- creación, lineamientos. Artes Visuales- Dirección de Artes del Ministerio de Cultura.

MinEducación. Ministerio de Educación de Colombia. (2013). Memorias del evento. Valoración de los procesos de creación artística y cultural en el marco de la acreditación de programas. Bogotá, 26 y 27 de junio de 2013. Casas Figueroa, M.V. (Comp.). Bogotá: SECAB-publicaciones.

Morales, B. L., Giraldo, A. M., Rodríguez, Y., \& Casas, P. (2018). Investigación-creación en los trabajos de grado de pregrado en música. Un estudio de caso. Revista Electrónica Complutense de Investigación en Educación Musical,(15), 97-118. DOI:https://doi.org/10.5209/RECIEM.59378.

Nancy, J-L. (2007). 58 indicios sobre el cuerpo Extensión del alma (D. Alvarado, Trad.). Buenos Aires: Ediciones La Cebra.

Orrantia, M. (2012). La escritura creativa en Colombia. Literatura: teoría, historia, crítica, 14(1), 287301. Bogotá: Universidad Nacional de Colombia.

Páramo, P. (Comp.). (2013). La investigación en ciencias sociales: estrategias de investigación. Bogotá: Universidad Piloto de Colombia.

Peña, I. (2014). El universo de la creación narrativa. Bogotá: Ediciones El Huaco.

Richardson, L. (1989). Writing A Method of Inquiry. En Handbook of Qualitative Research (pp. 516 529). EE. UU.: Texas A\&M University.

Silva-Cañaveral, S.J. (2016). La investigación-creación en el contexto de la formación doctoral en diseño y creación en Colombia. Revista de investigación, desarrollo e innovación, 7(1), 49-61. Doi: https://doi.org/10.19053/20278306.v7.n1.2016.5601.

Sloterdijk, P. (2006). Venir al mundo, venir al lenguaje. Lecciones de Frankfurt. Valencia: Pretexto.

Suescún, P. (2012). El artista y la escritura, posturas. En Una mirada al pensamiento del artista en la Universidad Nacional de Colombia (pp. 41-43). Bogotá: Universidad Nacional de Colombia.

Todorov, I. (1998). La conquista de América el problema del otro. México: Siglo XXI. 